Research Article

\title{
2-Restricted Edge Connectivity of Wheel Networks
}

\author{
Wei Feng $\mathbb{D}^{1,2}$ and Shiying Wang ${ }^{1}$ \\ ${ }^{1}$ College of Mathematics and Information Science, Henan Normal University, Xinxiang, Henan 453007, China \\ ${ }^{2}$ Institute of Discrete Mathematics, College of Mathematics and Physics, Inner Mongolia University for Nationalities, \\ Tongliao 028043, China \\ Correspondence should be addressed to Wei Feng; fengwei0422@sina.com
}

Received 26 April 2021; Accepted 26 June 2021; Published 6 July 2021

Academic Editor: Qiuhong Wu

Copyright ( 2021 Wei Feng and Shiying Wang. This is an open access article distributed under the Creative Commons Attribution License, which permits unrestricted use, distribution, and reproduction in any medium, provided the original work is properly cited.

\begin{abstract}
The $g$-restricted edge connectivity $(g$-REC) is an efficient index in evaluating the reliability and fault tolerance of large-scale processing systems. Assume that $G=(V, E)$ is a simple connected graph and $F \subseteq E$. The $g$-REC of $G$, denoted by $\lambda_{g}(G)$, is the minimum $|F|$ such that $G-F$ is disconnected and any component has at least $g$ nodes. The $n$-dimensional wheel network $\mathrm{CW}_{n}$, a kind of Cayley graph, possesses many desired features. In this paper, we establish that $\lambda_{2}\left(\mathrm{CW}_{n}\right)$ is $4 n-6$ for $n \geq 5$, and $\mathrm{CW}_{n}$ is $\lambda_{2}$ optimal.
\end{abstract}

\section{Introduction}

With the increase of interconnection networks' scale, the probability of processors and/or links' failing increases. How to evaluate the reliability and fault tolerance of a large-scale processing system has become a significant research topic. A graph $G=(V, E)$ can be always used to represent a practical interconnection network, where $V$ (resp. E) represents the set of all processors (resp. links). One of the classical measurements for evaluating the reliability and fault tolerance of a network is edge connectivity $\lambda(G)$ of a graph $G$. Generally, the larger the $\lambda(G)$ is, the more reliable the responding network is. However, this index has its shortcoming, that is, it tacitly supposes that each edge incidents with the same node in $G$ may fail simultaneously. It is very unlikely in the process of applications of large-scale networks.

One of methods for compensating this deficiency is to increase certain conditions in each component of $G-S$ in the concept of $\lambda(G)$. The $g$-restricted edge connectivity ( $g$-REC) was introduced by Fàbrega and Fiol [1]. Assume that $G=(V, E)$ is a simple connected graph and $F \subseteq E$. The $g$-REC of $G$, denoted by $\lambda_{g}(G)$, is the minimum $|F|$ such that $G-F$ is disconnected and any component has at least $g$ nodes. Clearly, if $G \neq K_{1}$, then $\lambda_{1}(G)=\lambda(G)$. In particular,
$\lambda_{2}(G)$ is equal to the conditional edge connectivity $\lambda^{\prime}(G)$ introduced in [2]. In [3], it is shown that the larger $\lambda_{2}(G)$ is, the more reliable the corresponding network is. Esfahanian et al. [2] have established that $\lambda_{2}(G)$ can be found by solving no more than $(\Delta \delta+|E(G)|-2)$ network flow problems and $\lambda(G) \leq \lambda_{2}(G) \leq \xi(G) \quad$ if $\quad \lambda_{2}(G)$ exists, where $\xi(G)=\min \{d(x)+d(y)-2: x y \in E(G)\}$. A graph $G$ with $\lambda_{2}(G)=\xi(G)$ is called a $\lambda_{2}$-optimal graph. 2-REC and $\lambda_{2}$-optimal graph have been discussed by many researchers $[4-8]$.

\section{Preliminaries}

2.1. Terminology and Notations. For those terminology and notations not stated here, the readers can refer to $[9,10]$. Suppose that $G=(V, E)$ is an undirected and simple graph. For any node $u \in V(G)$, define $N_{G}(u)=\{v \in V(G)$ : $u v \in E(G)\}$ (resp. $\mathrm{NE}_{G}(u)=\{e \in E(G) \mid e$ is incident with $u$ in $G$ ) to be the neighborhood (resp. edge neighborhood) of $u$ in $G$. The degree $d_{G}(u)$ of a node $u$ is $\left|N_{G}(u)\right| . \delta(G)=$ $\min \{d(u) \mid u \in V\} \quad$ (resp. $\Delta(G)=\max \{d(u) \mid u \in V\}$ ) is the minimum (resp. maximum) degree of a graph $G$. For any $W \subseteq V$, let $N_{G}(W)=\left(\cup_{u \in W} N_{G}(u)\right) \backslash W$. The length of a shortest cycle of $G$ is defined as the girth of $G$. For $X \subset V$, let $G[X]$ represent the induced subgraph of $X$ in $G$. For 
$X, Y \subseteq V(G)$, let $[X, Y]=\{x y: x \in X, y \in Y$ and $x y \in E(G)$. If $d_{G}(u)=k$, for any $u \in V(G)$, then the graph $G$ is $k$-regular. The subscript $G$ of $N_{G}(u), \mathrm{NE}_{G}(u), d_{G}(u)$, and $N_{G}(W)$ are usually omitted if $G$ is already known from the context. A graph $G$ is node transitive if there exist $\tau \in \Gamma(G)$ such that $\tau(x)=y$, for $\forall x, y \in V(G)$, where $\Gamma(G)$ is the automorphism group of $G$. Considering two graphs $G_{1}$ and $G_{2}, G_{1} \cong G_{2}$ means that they are isomorphic.

2.2. The Wheel Networks. Assume that $Q$ is a finite group. Suppose that a spanning set $S$ of $Q$ satisfies $I \notin S$, where $I$ is the identity element of $Q$. A directed Cayley graph Cay $(S, Q)$ can be stated as follows: its node set is $Q$, and its directed arc set is $\{(q, q s): q \in Q, s \in S\}$. If $s^{-1} \in S$ holds for $\forall s \in S$, then Cay $(S, Q)$ is an undirected graph.

Assume that $H$ is a connected and simple graph and $V(H)=\{1,2, \ldots, n\}(n \geq 3)$. If we consider each edge in $H$ as a transposition of the symmetric graph $S_{n}$, then the edge set of $H$ can correspond exactly to a transposition set $S$ in $S_{n}$. From this sense, $H$ is usually called a transposition simple graph. The Cayley graph Cay $(S,\langle S\rangle)$ is called the corresponding Cayley graph of $H$, also denoted by Cay $(H,\langle S\rangle)$. By Akers et al. [11], $\langle S\rangle=S_{n}$. If $H$ is a tree (resp. path, star), then the corresponding Cayley graph, denoted by $C \Gamma_{n}$ (resp. $\left.B_{n}, S_{n}\right)$ is called a transposition tree (resp. bubble-sort graph, star graph) [11]. If $H=\mathrm{SE}_{n}(n \geq 3)$, where $V\left(\mathrm{SE}_{n}\right)=\{1,2, \ldots, n\}$ and $E\left(\mathrm{SE}_{n}\right)=$ $\{(1, i): 2 \leq i \leq n\} \cup\{(i, i+1): 2 \leq i \leq n-1\}$, then the corresponding Cayley graph Cay $\left(\mathrm{SE}_{n}, S_{n}\right)$, denoted by $\mathrm{BS}_{n}$, is called a bubble-sort star graph [12]. If $H=W_{n}(n \geq 4)$, where $V\left(W_{n}\right)=$ $\{1,2, \ldots, n\} \quad$ and $E\left(W_{n}\right)=\{(1, i): 2 \leq i \leq n\} \cup$ $\{(i, i+1): 2 \leq i \leq n-1\} \cup\{(2, n)\}$, then the corresponding Cayley graph Cay $\left(W_{n}, S_{n}\right)$, denoted by $\mathrm{CW}_{n}$, is called a $n$-dimensional wheel network [13]. In other words, $V\left(\mathrm{CW}_{n}\right)=S_{n}$. And, for any $u, v \in V\left(\mathrm{CW}_{n}\right), u v \in E\left(\mathrm{CW}_{n}\right)$ iff $u=v(1, i), \quad 2 \leq i \leq n, \quad$ or $\quad u=v(i, i+1), \quad 2 \leq i \leq n-1$, or $u=v(2, n)$. The diameters of $\mathrm{BS}_{n}$ and $\mathrm{CW}_{n}$ are both $\lfloor 3(n-1) / 2\rfloor$, where $n \geq 7[12,14] . \mathrm{BS}_{n}$ can be embedded in a $\mathrm{CW}_{n} . \mathrm{CW}_{n}$ surpasses $\mathrm{BS}_{n}$ in connectivity $[15,16]$. Hence, $\mathrm{CW}_{n}$ is also a desirable processing model. $\mathrm{CW}_{n}$ is studied by many researchers $[13,14,17-25] . \mathrm{CW}_{n}$ for $n=4$ has been depicted in Figure 1.

The permutation $\left(\begin{array}{cccc}1 & 2 & \cdots & n \\ p_{1} & p_{2} & \cdots & p_{n}\end{array}\right)$ can be conveniently denoted by $p_{1} p_{2} \cdots p_{n}$. Let $\tau$ and $\sigma$ be two permutations. The product $\sigma \tau$ means the composition function $\tau$ followed by $\sigma$, for example, $(12)(13)=(132)$.

If we delete the node labeling $n$ in the transposition simple graph of $\mathrm{CW}_{n}$, then a graph $\mathrm{SE}_{n-1}$ on node set $\{1,2, \ldots, n-1\}$ is obtained. Note that the Cayley graph responding $\mathrm{SE}_{n-1}$ is $\mathrm{BS}_{n-1}$. Hence, $\mathrm{CW}_{n}$ can be decomposed into $n$ copies of $\mathrm{BS}_{n-1}$ as follows. For any $i \in\{1,2, \ldots, n\}$, suppose that $\mathrm{CW}_{n}^{i}$ is the subgraph of $\mathrm{CW}_{n}$ induced by the following node set $\left\{u_{1} u_{2}, \ldots, u_{n-1} i \mid u_{1} u_{2}, \ldots, u_{n-1}\right.$ ranges over any permutations of $\{1,2, \ldots, n\} \backslash\{i\}$. Hence, $\mathrm{CW}_{n}^{i} \cong \mathrm{BS}_{n-1}$. For any $v \in V\left(\mathrm{CW}_{n}^{i}\right)$, then three neighbors $v(1 n), v(n-1, n)$, and $v(2 n)$ of $v$ are all called outside neighbors of $v$, denoted by $v^{+}=v(1 n), v^{-}=v(n-1, n)$, and $v^{*}=v(2 n)$, respectively. An edge $u v$ is called a cross-edge in the given decomposition if $u, v$ are in distinct $\mathrm{CW}_{n}^{i}$ s.

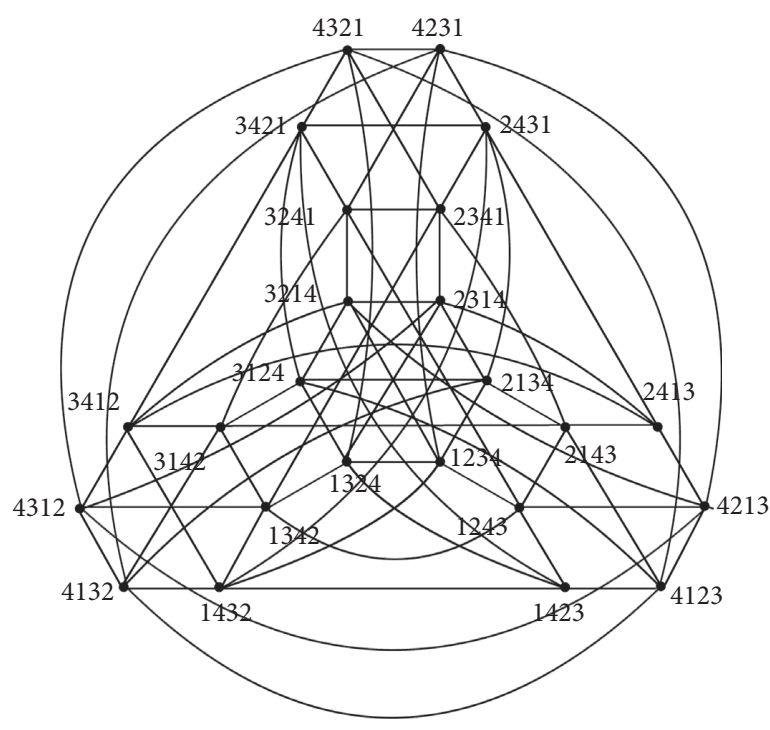

Figure 1: The wheel network $\mathrm{CW}_{4}$.

Proposition 1 (see [9]). $\kappa(G) \leq \lambda(G) \leq \delta(G)$.

Lemma 1 (see [15]). $\kappa\left(B S_{n}\right)=2 n-3, \forall n \geq 4$.

Proposition 2 (see [12]). $B S_{n}$ is (2n-3)-regular, $\forall n \geq 4$.

Proposition 3 (see [26]). $\forall n \geq 4, C W_{n}$ is (2n-2)-regular, node transitive.

Proposition 4 (see [26]). $\forall n \geq 4, C W_{n}$ is bipartite.

Proposition 5 (see [16]). $\forall v \in V\left(C W_{n}^{i}\right)$, where $i \in\{1,2, \ldots, n\}$. Then, $v^{+}, v^{-}$, and $v^{*}$ belong to 3 distinct $C W_{n}^{j} s(j \neq i)$.

Proposition 6 (see [16]). ie number of independent crossedges between two distinct $C W_{n}^{i}$ 's is $3(n-2)$ !.

Proposition 7 (see [27]). For $u, v \in V\left(C W_{n}^{i}\right)$, where $i \in\{1,2, \ldots, n\}$, then $\left\{u^{+}, u^{-}, u^{*}\right\} \cap\left\{v^{+}, v^{-}, v^{*}\right\}=\varnothing$.

Lemma 2 (see [2]). $\lambda(G) \leq \lambda_{2}(G) \leq \xi(G)$.

\section{2-REC of $n$-Dimensional Wheel Network $\lambda_{2}\left(\mathbf{C W}_{n}\right)$}

Theorem 1. $\lambda_{2}\left(C W_{n}\right)=4 n-6$, for $n \geq 4$, and $C W_{n}$ is a $\lambda_{2}$-optimal graph.

Proof. By Propositions 3 and 4, we can obtain $\xi\left(\mathrm{CW}_{n}\right)=4 n-6$. By Lemma $2, \lambda_{2}\left(\mathrm{CW}_{n}\right) \leq 4 n-6$, for $n \geq 4$. Next, we prove $\lambda_{2}\left(\mathrm{CW}_{n}\right) \geq 4 n-6$, for $n \geq 4$.

We decompose $\mathrm{CW}_{n}$ into $n$ disjoint $\mathrm{BS}_{n-1}$ copies $\mathrm{CW}_{n}^{1}, \mathrm{CW}_{n}^{2}, \ldots, \mathrm{CW}_{n}^{n}$ as above, where each $\mathrm{CW}_{n}^{i} \cong \mathrm{BS}_{n-1}$. Assume that $F$ is any edge subset of $\mathrm{CW}_{n}$ with $|F| \leq 4 n-7$ such that $\mathrm{CW}_{n}-F$ has no isolated nodes. We just need to prove that $\mathrm{CW}_{n}-F$ is connected. Assume that $F_{i}=F \cap E\left(\mathrm{CW}_{n}^{i}\right), \quad E^{[i, j]}=\left[V\left(\mathrm{CW}_{n}^{i}\right), V\left(\mathrm{CW}_{n}^{j}\right)\right], \quad$ and 
$F^{[i, j]}=E^{[i, j]} \cap F$. Clearly, $F=\left(\cup_{i=1}^{n} F_{i}\right) \cup\left(\cup_{1 \leq i \neq j \leq n} F^{[i, j]}\right)$. We partition $\mathrm{CW}_{n}$ into two parts: $S_{1}$ and $S_{2}$, where $S_{1}=$ $\left\{\mathrm{CW}_{n}^{i}:\left|F_{i}\right| \geq 2 n-5\right\}$ and $S_{2}=\left\{\mathrm{CW}_{n}^{i}:\left|F_{i}\right| \leq 2 n-6\right\}$. Obviously, $\left|S_{1}\right|+\left|S_{2}\right|=n$. Let $\widetilde{G}=\mathrm{CW}_{n}\left[V\left(\cup_{\mathrm{CW}_{n}^{i} \in S_{2}} \mathrm{CW}_{n}^{i}\right)\right]-F$. We distinguish two cases for $n$.

Case 1. $n \geq 5$.

Claim 1. If $S_{2} \neq \varnothing$, then $\widetilde{G}$ is connected.

Proof. Note that $F_{i}$ corresponding to $\mathrm{CW}_{n}^{i}$ in $S_{2}$ satisfies that $\left|F_{i}\right| \leq 2 n-6$. By Propositions 1 and 2 and Lemma 1 , $\lambda\left(\mathrm{CW}_{n}^{i}\right)=\lambda\left(\mathrm{BS}_{n-1}\right)=2(n-1)-3=2 n-5$, for $i \in\{1,2$, $\ldots, n\}$. Hence, every $\mathrm{CW}_{n}^{i}-F_{i}$ is connected, for any $\mathrm{CW}_{n}^{i}$ in $S_{2}$. By Proposition 6 and $3(n-2) !>4 n-7 \geq|F|$ for $n \geq 5$, hence, $\mathrm{CW}_{n}^{i}-F_{i}$ is connected to $\mathrm{CW}_{n}^{j}-F_{j}$ for any two distinct $\mathrm{CW}_{n}^{i}$ and $\mathrm{CW}_{n}^{j}$ in $S_{2}$. We deduce that $G$ is connected. Claim 1 is completed.

Since $3 \times(2 n-5)>4 n-7 \geq|F|$, for $n \geq 5$, we have that $\left|S_{1}\right| \leq 2$. We prove that $\mathrm{CW}_{n}-F$ is connected in the following situations.

Case 1.1. $\left|S_{1}\right|=0,\left|S_{2}\right|=n$.

Using a proof similar to that in Claim 1, we can yield that $\mathrm{CW}_{n}-F=\widetilde{G}$ is connected.

Case 1.2. $\left|S_{1}\right|=1,\left|S_{2}\right|=n-1$.

Let $\mathrm{CW}_{n}^{i}$ be the only one $\mathrm{BS}_{n-1}$ copy satisfying $\left|F_{i}\right| \geq 2 n-5$. Let $C$ be any component of $\mathrm{CW}_{n}^{i}-F_{i}$. Next, we prove that $C$ is connected to $G$. Note that $C_{n}-F$ has no isolated nodes. If $C$ contains only a node $u$, then $u$ is connected to $\widetilde{G}$ by one of $u u^{+}, u u^{-}$, and $u u^{*}$. If $|C| \geq 2$, then $C$ has an edge $u v \in\left(E\left(\mathrm{CW}_{n}^{i}\right)-F\right)$. Let $N_{\mathrm{CW}_{n}^{i}}(u)=\left\{u_{1}, u_{2}, \ldots, u_{2 n-6}, v\right\} \quad$ and $\quad N_{\mathrm{CW}_{n}^{i}}(v)=\left\{v_{1}, v_{2}\right.$, $\left.\ldots, v_{2 n-6}, u\right\}$. By Propositions 5 and 7, there is a path $P$ in $\left\{u u_{i} u_{i}^{+}, u u_{i} u_{i}^{-}, u u_{i} u_{i}^{*}, v v_{i} v_{i}^{+}, \quad v v_{i} v_{i}^{-}, v v_{i} v_{i}^{*}\right\} \bigcup\left\{u u^{+}, u u^{-}\right.$, $\left.u u^{*}, v v^{+}, v v^{-}, v v^{*}\right\}$ such that $E(P) \cap F=\varnothing$. So, $C$ is connected to $\widetilde{G}$ by $P$. We deduce that $\mathrm{CW}_{n}-F$ is connected.

Case 1.3. $\left|S_{1}\right|=2$ and $\left|S_{2}\right|=n-2$.

Let $\mathrm{CW}_{n}^{i}, \mathrm{CW}_{n}^{j}$ be just the two $\mathrm{BS}_{n-1}$ copies satisfying $\left|F_{i}\right|,\left|F_{j}\right| \geq 2 n-5$. It is easy to see that $2 n-5$ $\leq\left|F_{i}\right|,\left|F_{j}\right| \leq 4 n-7-(2 n-5)=2 n-2$ and $\left|F \backslash F_{i} \backslash F_{j}\right| \leq$ $4 n-7-2(2 n-5)=3$. Note that $\left|F_{i}\right|+\left|F_{k}\right|+\left|F^{[i, k]}\right| \leq 2 n-$ $2+3=2 n+1<\left|E^{[i, j]}\right|=3(n-2)$ ! for $n \geq 5$, where $\mathrm{CW}_{n}^{k}$ corresponding to $F_{k}$ belongs to $S_{2}$. We have that $\mathrm{CW}_{n}^{i}-F_{i}$ and $\mathrm{CW}_{n}^{k}-F_{k}$ are connected. Similarly, we can deduce that $\mathrm{CW}_{n}^{j}-F_{j}$ and $\mathrm{CW}_{n}^{k}-F_{k}$ are connected. By Claim $1, \mathrm{CW}_{n}-$ $F$ is connected.

Case 2. $n=4$.

Since $4 \times(2 n-5)>4 n-7 \geq|F|$ for $n=4$, we have that $\left|S_{1}\right| \leq 3$. We prove that $\mathrm{CW}_{4}-F$ is connected in the following cases:

Case 2.1. $\left|S_{1}\right|=0$ and $\left|S_{2}\right|=4$.

Using a proof similar to that in Claim 1, we can deduce that $\mathrm{CW}_{4}^{i}-F_{i}$ is connected for $1 \leq i \leq 4$. We say that $\widetilde{G}$ is connected. Otherwise, there are $\mathrm{CW}_{4}^{i}-F_{i}$ and $\mathrm{CW}_{4}^{j}-F_{j}$, and they are disconnected, where $\mathrm{CW}_{4}^{i}-F_{i}$ and $\mathrm{CW}_{4}^{j}-F_{j}$ are both in $S_{2}$. By Proposition 6, $\left|F^{[i, j]}\right|=\left|E^{[i, j]}\right|=3(n-2) !=3 \times(4-2) !=6$. Note that $|F| \leq 4 n-7=4 \times 4-7=9$. Hence, there is a $\mathrm{BS}_{4-1}$ copy $\mathrm{CW}_{4}^{k}$ in $S_{2}$ such that $\left|F^{[i, k]}\right|<\left|E^{[i, k]}\right|$ and $\left|F^{[j, k]}\right|<\left|E^{[j, k]}\right|$, where $k \in\{1,2,3,4\} \backslash\{i, j\}$. We obtain that $\mathrm{CW}_{4}^{i}-F_{i}$ and $\mathrm{CW}_{4}^{j}-F_{j}$ are both connected to $\mathrm{CW}_{4}^{k}-F_{k}$. Therefore, we have that $\mathrm{CW}_{4}-F$ is connected.

Case 2.2. $\left|S_{1}\right|=1$ and $\left|S_{2}\right|=3$.

Let $\mathrm{CW}_{4}^{i}$ be the only one $\mathrm{BS}_{4-1}$ copy satisfying $\left|F_{i}\right| \geq 2 n-5=2 \times 4-5=3$. Using a proof similar to that in Claim 1, we can include that $\mathrm{CW}_{4}^{j}-F_{j}$ is connected for each $\mathrm{CW}_{4}^{j}$ in $S_{2}$. We say that $\widetilde{G}$ is connected. Otherwise, there are $\mathrm{CW}_{4}^{j}-F_{j}$ and $\mathrm{CW}_{4}^{k}-F_{k}$ in $S_{2}$, and they are disconnected in $\mathrm{CW}_{4}$. By Proposition 6, we have that $\left|F^{[j, k]}\right|=\left|E^{[j, k]}\right|=3(n-2) !=3 \times(4-2) !=6$. Note that $\left|F \backslash F_{i}\right| \leq 6$ holds clearly. Hence, there is a $\mathrm{BS}_{4-1}$ copy $\mathrm{CW}_{4}^{l}$ in $S_{2}$ such that $\left|F^{[j, l]}\right|<\left|E^{[j, l]}\right|$ and $\left|F^{[k, l]}\right|<\left|E^{[k, l]}\right|$, where $l \in\{1,2,3,4\} \backslash\{i, j, k\}$. We obtain that $\mathrm{CW}_{4}^{j}-F_{j}$ and $\mathrm{CW}_{4}^{k}-$ $F_{k}$ are both connected to $\mathrm{CW}_{4}^{l}-F_{l}$. Therefore, we have that $\widetilde{G}$ is connected.

Let $C$ be any component of $\mathrm{CW}_{4}^{i}-F_{i}$. Next, we prove that $C$ is connected to $\widetilde{G}$. If $C$ contains only a node $u$, then $u$ is connected to $\widetilde{G}$ by one of $u u^{+}, u u^{-}$, and $u u^{*}$, since $\mathrm{CW}_{4}-$ $F$ has no isolated nodes. If $|C| \geq 2$, then $C$ has an edge $u v \in\left(E\left(\mathrm{CW}_{n}^{i}\right)-F\right)$. Let $\quad N_{\mathrm{CW}_{4}^{i}}(u)=\left\{u_{1}, u_{2}, v\right\} \quad$ and $N_{\mathrm{CW}_{4}^{i}}(v)=\left\{v_{1}, v_{2}, u\right\}$. By Propositions 5 and 7 , there is a path $P$ in $\left\{u u_{i} u_{i}^{+}, u u_{i} u_{i}^{-}, u u_{i} u_{i}^{*}, v v_{i} v_{i}^{+}, v v_{i}\right.$ $\left.v_{i}^{-}, v v_{i} v_{i}^{*}\right\} \bigcup\left\{u u^{+}, u u^{-}, u u^{*}, v v^{+}, v v^{-}, v v^{*}\right\} \quad$ such that $E(P) \cap F=\varnothing$, where $i \in\{1,2\}$. So, $C$ is connected to $\widetilde{G}$ by $P$. We deduce that $\mathrm{CW}_{4}-F$ is connected.

Case 2.3. $\left|S_{1}\right|=2$ and $\left|S_{2}\right|=2$.

Let $\mathrm{CW}_{4}^{i}, \mathrm{CW}_{4}^{j}$ be just the two $\mathrm{BS}_{4-1}$ copies satisfying $\left|F_{i}\right|,\left|F_{j}\right| \geq 2 n-5=2 \times 4-5$. Using a proof similar to that in Claim 1, we include that $\mathrm{CW}_{4}^{k}-F_{k}$ is connected for each $\mathrm{CW}_{4}^{k} \quad$ in $\quad S_{2}$. Clearly, $\quad\left|F \backslash F_{i} \backslash F_{j}\right| \leq 4 n-7-$ $2(n-5)=3<3(4-2) !=\left|E^{[k, l]}\right|, \quad$ where $\quad k, l \in\{1,2,3,4\}$ $\checkmark\{i, j\}$. By Proposition 6, $\widetilde{G}$ is connected.

Case 2.3.1. Both $\mathrm{CW}_{4}^{i}-F_{i}$ and $\mathrm{CW}_{4}^{j}-F_{j}$ are connected.

We claim that $C W_{4}-F$ is connected. Otherwise, there are two copies $\mathrm{CW}_{4}^{k}$ and $C W_{4}^{l}$ are disconnected for $1 \leq k \neq l \leq 4 . \quad$ By $\quad$ Proposition $\quad 6, \quad\left|E^{[k, l]}\right|=$ $3(n-2) !=3 \times(4-2) !=6$. Note that $|F| \leq 4 n-7$ $=4 \times 4-7=9$. Hence, for any $\mathrm{CW}_{4}^{m}, m \in\{1,2,3,4\} \backslash\{k, l\}$ such that both $\mathrm{CW}_{4}^{k}-F_{k}$ and $\mathrm{CW}_{4}^{l}-F_{l}$ are connected to $\mathrm{CW}_{4}^{m}-F_{m}$. We deduce that $\mathrm{CW}_{4}-F$ is connected.

Case 2.3.2. Only one of $\mathrm{CW}_{4}^{i}-F_{i}$ and $\mathrm{CW}_{4}^{j}-F_{j}$ is connected.

Assume that $\mathrm{CW}_{4}^{i}-F_{i}$ be disconnected and $\mathrm{CW}_{4}^{j}-F_{j}$ be connected. By Proposition 6 and $\left|F \backslash F_{1} \backslash F_{2}\right| \leq 3<6=\left|E^{[j, k]}\right|, \quad \mathrm{CW}_{4}^{j}-F_{j}$ is connected to $\mathrm{CW}_{4}^{k}-F_{k}$, where $\mathrm{CW}_{4}^{k}$ is any one in $S_{2}$. Therefore, $\mathrm{CW}_{4}^{j}-F_{j}$ is connected to $\widetilde{G}$. Let $C$ be any component of $C_{4}^{i}-F_{i}$. Next, we prove that $C$ is connected to $\widetilde{G}$. If $C$ contains only a node $u$, then $u$ is connected to $\widetilde{G}$ by one of $u u^{+}, u u^{-}$, and 
$u u^{*}$, since $\mathrm{CW}_{4}-F$ has no isolated nodes. If $|C| \geq 2$, then $C$ has an edge $u v \in\left(E\left(\mathrm{CW}_{4}^{i}\right)-F\right)$. Let $N_{\mathrm{CW}_{4}^{i}}(u)=\left\{u_{1}, u_{2}, v\right\}$ and $N_{\mathrm{CW}_{n}^{i}}(v)=\left\{v_{1}, v_{2}, u\right\}$. By Propositions 5 and 7 , there is a path $P$ in $\left\{u u_{i} u_{i}^{+}, u u_{i} u_{i}^{-}, u u_{i} u_{i}^{*}, v v_{i} v_{i}^{+}, v v_{i} v_{i}^{-}\right.$, $\left.v v_{i} v_{i}^{*}\right\} \bigcup\left\{u u^{+}, u u^{-}, u u^{*}, v v^{+}, v v^{-}, v v^{*}\right\}$ such that $E(P) \cap F=\varnothing$, where $i \in\{1,2\}$. So, $C$ is connected to $\widetilde{G}$ by $P$. We deduce that $\mathrm{CW}_{4}-F$ is connected.

Case 2.3.3. Neither $\mathrm{CW}_{4}^{i}-F_{i}$ nor $\mathrm{CW}_{4}^{j}-F_{j}$ is connected.

Let $C_{i 1}, C_{i 2}, \ldots, C_{i k}$ be all components in $\mathrm{CW}_{4}^{i}-F_{i}$, and let $C_{j 1}, C_{j 2}, \ldots, C_{j l}$ be all components in $C^{j} W_{4}^{j}-F_{j}$. We consider any $C_{i s}$ for $s \in\{1,2, \ldots, k\}$. Let $u \in V\left(C_{i s}\right)$, and we give Claim 2 as follows:

Claim 2. $u$ is connected to $\widetilde{G}$.

Proof. By Proposition 5, it is easy to prove that, at most one cross-edge of $\left\{u u^{+}, u u^{-}, u u^{*}\right\}$ is in $E^{[i, j]}$, where $j \in\{1,2,3,4\} \backslash\{i\}$. We distinguish two cases to complete Claim 2.

Case 2.3.3.1. $\left|\left\{u u^{+}, u u^{-}, u u^{*}\right\} \cap E^{[i, j]}\right|=0$.

If $\left\{u u^{+}, u u^{-}, u u^{*}\right\} \subseteq F$, then it is a contradiction to that $\mathrm{CW}_{4}-F$ has no isolated nodes. Hence, there is an element $e \in\left\{u u^{+}, u u^{-}, u u^{*}\right\}$ and $e \notin F$. By Proposition $5, u$ is connected to $\widetilde{G}$ by e. Claim 2 is completed in this case.

Case 2.3.3.2. $\left|\left\{u u^{+}, u u^{-}, u u^{*}\right\} \cap E^{[i, j]}\right|=1$.

Suppose that $u u^{+} \in E^{[i, j]}$. Since $\left|F_{1}\right|,\left|F_{2}\right| \geq 2 n-5=2 \times$ $4-5$ and $|F| \leq 4 n-7=4 \times 4-7=9$, we can deduce $\left|F \backslash F_{1} \backslash F_{2}\right| \leq 4 n-7-(2 n-5)-(2 n-5)=3$. Note that $\left|\left\{u u^{+}, u u^{-}, u u^{*}, u^{+}\left(u^{+}\right)^{+}, u^{+}\left(u^{+}\right)^{-}, u^{+}\left(u^{+}\right)^{*}\right\}\right|=6$ by Proposition 5. So, there is an element $e \in\left\{u u^{+}, u u^{-}, u u^{*}, u^{+}\left(u^{+}\right)^{+}, u^{+}\left(u^{+}\right)^{-}, u^{+}\left(u^{+}\right)^{*}\right\}$ and $e \notin F$ such that $u$ is connected to $\widetilde{G}$ by $e$. The situations of $u u^{-} \in E^{[i, j]}$ and $u u^{*} \in E^{[i, j]}$ can be similarly proved. Claim 2 is completed in this case.

Similarly, we can deduce that a node $v$ in any $C_{j t}$ for $t \in\{1,2, \ldots, l\}$ is connected to $\widetilde{G}$. By the arbitrariness of $C_{i s}$ and $C_{j t}$, we prove that each of $C_{i 1}, C_{i 2}, \ldots, C_{i k}, C_{j 1}, C_{j 2}, \ldots, C_{j l}$ is connected to $\widetilde{G}$. So, $\mathrm{CW}_{4}-F$ is connected.

Case 2.4. $\left|S_{1}\right|=3$ and $\left|S_{2}\right|=1$.

Let $\mathrm{CW}_{4}^{i}, \mathrm{CW}_{4}^{j}, \mathrm{CW}_{4}^{k}$ be just the three $\mathrm{BS}_{4-1}$ copies satisfying $\left|F_{i}\right|,\left|F_{j}\right|,\left|F_{k}\right| \geq 2 n-5=2 \times 4-5=3$. It implies that $\left|F_{i}\right|=\left|F_{j}\right|=\left|F_{k}\right|=3$ since $|F| \leq 4 n-7=4 \times 4-7=9$. Hence, there is no edges of $F$ in $E\left(\mathrm{CW}_{4}\right) \backslash E\left(\mathrm{CW}_{4}^{i}\right) \backslash E\left(\mathrm{CW}_{4}^{j}\right) \backslash E\left(\mathrm{CW}_{4}^{k}\right)$. It implies that $F \cap F^{[i, j]}=\varnothing$, for $1 \leq i \neq j \leq 4$, and $\left|F_{l}\right|=0$, for $l \in\{1,2,3,4\} \backslash\{i, j, k\}$. Note that $\left|F_{i}\right|+\left|F_{l}\right|+\left|F^{[i, l]}\right|=$ $3<6=3(n-2) !=\left|E^{[i, l]}\right|$. Hence, $\mathrm{CW}_{4}^{j}-F_{j}$ and $\mathrm{CW}_{4}^{l}-F_{l}$ are connected. Similarly, both $\mathrm{CW}_{4}^{j}-F_{j}$ and $\mathrm{CW}_{4}^{k}-F_{k}$ are connected to $\mathrm{CW}_{4}^{l}-F_{l}$. We have that $\mathrm{CW}_{4}-F$ is connected.

Therefore, we established $\lambda_{2}\left(\mathrm{CW}_{n}\right)=4 n-6$ for $n \geq 4$. Since $\lambda_{2}\left(\mathrm{CW}_{n}\right)=\xi\left(\mathrm{CW}_{n}\right)$, we say that $\mathrm{CW}_{n}$ is a $\lambda_{2}$-optimal graph. The theorem is completed.

\section{Conclusion}

The $g$-restricted edge connectivity is a generalization of classical edge connectivity. It is a more refined index in measuring fault tolerance of interconnection networks. Here, we obtain that 2-restricted edge connectivity of wheel network $\mathrm{CW}_{n}$. Note that wheel network $\mathrm{CW}_{n}$ is a kind of regular graph. A nontrivial graph with order $n$ whose degree set contains $n-1$ elements is named an antiregular graph [28]. It would be interesting to find the $g$-restricted edge connectivity of antiregular graphs.

\section{Data Availability}

The data used to support the findings of the study are available from the corresponding author upon request.

\section{Conflicts of Interest}

The authors declare that they have no conflicts of interest.

\section{Acknowledgments}

This work was supported by the NSFC (61772010), the Higher Educational Scientific Research Projects of Inner Mongolia (NJZY21439), the project of IMUN (NMDGP17106), and the 2020 Scientific Research Project for Postgraduates of Henan Normal University (YL202008).

\section{References}

[1] J. Fàbrega and M. A. Fiol, "Extraconnectivity of graphs with large girth,” Discrete Mathematics, vol. 127, pp. 163-170, 1994.

[2] A.-H. Esfahanian and S. L. Hakimi, "On computing a conditional edge connectivity of a graph," Information Processing Letters, vol. 27, no. 4, pp. 195-199, 1988.

[3] M. Wang and Q. Li, "Conditional edge connectivity properties, reliability comparisons and transitivity of graphs," Discrete Mathematics, vol. 258, no. 1-3, pp. 205-214, 2002.

[4] Q. Li and Q. Li, "Reliability analysis of circulants," Networks, vol. 31, no. 2, pp. 61-65, 1998.

[5] Q. L. Li and Q. Li, "Super edge connectivity properties of connected edge symmetric graphs," Networks, vol. 33, pp. 147-159, 1999.

[6] J. Meng, "Optimally super-edge-connected transitive graphs," Discrete Mathematics, vol. 260, no. 1-3, pp. 239-248, 2003.

[7] J.-M. Xu and K.-L. Xu, "On restricted edge-connectivity of graphs,” Discrete Mathematics, vol. 243, no. 1-3, pp. 291-298, 2002.

[8] M. Xu and J. Jing, "The connectivity and super connectivity of bubble-sort graph," Acta Mathematicae Applicatae Sinica, vol. 35, no. 5, pp. 789-794, 2012, in Chinese.

[9] J. A. Bondy and U. S. R. Murty, Graph Theory, Springer, New York, NY, USA, 2007.

[10] W. Thomas, Hungerford, Algebra, Springer, New York, NY, USA, 1974.

[11] S. B. Akers and B. Krishnamurthy, "A group-theoretic model for symmetric interconnection networks," IEEE Transactions on Computers, vol. 38, no. 4, pp. 555-566, 1989.

[12] Z.-T. Chou, C.-C. Hsu, and J.-P. Sheu, "Bubble-sort star graphs: a new interconnection network," in Proceedings of the 
International Conference on Parallel and Distributed Systems, pp. 41-48, Tokyo, Japan, June 1996.

[13] H. Shi and J. Lu, "On conjectures of interconnection networks," Computer Engineering and Applications, vol. 44, no. 31, pp. 112-115, 2008, in Chinese.

[14] H. Shi, F. Hou, J. Ma, and G. Wang, "Study on diameter and average distance of wheel network," Journal of Gansu Sience, vol. 24, no. 2, pp. 103-106, 2012, in Chinese.

[15] H. Cai, H. Liu, and M. Lu, "Fault-tolerant maximal localconnectivity on bubble-sort star graphs," Discrete Applied Mathematics, vol. 181, pp. 33-40, 2015.

[16] W. Feng, Jirimutu, and S. Wang, "The nature diagnosability of wheel graph networks under the PMC model and $\mathrm{MM}^{*}$ model," ARS Combinatoria, vol. 143, pp. 255-287, 2019.

[17] Y. Wei and $\mathrm{M}$. Xu, "The 1, 2-good-neighbor conditional diagnosabilities of regular graphs," Applied Mathematics and Computation, vol. 334, pp. 295-310, 2018.

[18] J. Tu, Y. Zhou, and G. Su, "A kind of conditional connectivity of Cayley graphs generated by wheel graphs," Applied Mathematics and Computation, vol. 301, pp. 177-186, 2017.

[19] M.-M. Gu and R.-X. Hao, "Reliability analysis of Cayley graphs generated by transpositions," Discrete Applied Mathematics, vol. 244, pp. 94-102, 2018.

[20] X. Hu, Y. Tian, and J. Meng, "Super $R^{k}$-vertex-connectedness," Applied Mathematics and Computation, vol. 339, pp. 812-819, 2018.

[21] X. Hu, W. Yang, Y. Tian, and J. Meng, "Equal relation between g-good-neighbor diagnosability under the PMC model and g-good-neighbor diagnosability under the $\mathrm{MM}^{*}$ model of a graph," Discrete Applied Mathematics, vol. 262, pp. 96-103, 2019.

[22] Y. Wei and M. Xu, "Conditional diagnosability of Cayley graphs generated by wheel graphs under the PMC model," Theoretical Computer Science, vol. 849, pp. 163-172, 2021.

[23] W. Feng and S. Wang, "The diagnosability of wheel networks with missing edges under the comparison model," Mathematics, vol. 8, no. 10, p. 1818, 2020.

[24] W. Feng and S. Wang, "The 2-extra connectivity of wheel networks," Mathematical Problems in Engineering, vol. 2020, Article ID 8910240, 5 pages, 2020.

[25] W. Feng and S. Wang, "Structure connectivity and substructure connectivity of wheel networks," Theoretical Computer Science, vol. 850, pp. 20-29, 2021.

[26] F. Hou, Some New Results of the Wheel Networks and BubbleSort Star Networks, Northwest Normal University, Lanzhou, China, 2013, in Chinese.

[27] W. Feng, J. Ren, C. Enhe, and S. Wang, "The 2-good-neighbor connectivity of wheel graph networks," Utilitas Mathematica, vol. 116, pp. 139-167, 2020.

[28] A. Ali, "A survey of antiregular graphs," Contributions to Mathematics, vol. 1, pp. 67-79, 2020. 